Revue scientifique sur la conception et l'aménagement de l'espace

\title{
Le rôle des riverains dans le façonnement des interfaces ville-forêt
}

Représentations et pratiques dans les franges urbaines des Landes de Gascogne

The Role of Local Inhabitants in Shaping City-Forest Interfaces -

Representations and Practises on the Urban Fringes of the Landes de Gascogne

\section{Christine Bouisset et Isabelle Degrémont}

\section{(2) OpenEdition}

Édition électronique

URL : http://journals.openedition.org/paysage/10237

DOI : $10.4000 /$ paysage. 10237

ISSN : 1969-6124

Éditeur :

École nationale supérieure du paysage de Versailles-Marseille, Institut national des sciences appliquées Centre Val de Loire - École de la nature et du paysage, École nationale supérieure d'architecture et de paysage de Bordeaux, École nationale supérieure d'architecture et de paysage de Lille, Agrocampus Angers

\section{Référence électronique}

Christine Bouisset et Isabelle Degrémont, « Le rôle des riverains dans le façonnement des interfaces ville-forêt », Projets de paysage [En ligne], 13 | 2015, mis en ligne le 31 décembre 2015, consulté le 04 septembre 2020. URL : http://journals.openedition.org/paysage/10237 ; DOI : https://doi.org/ 10.4000/paysage.10237

Ce document a été généré automatiquement le 4 septembre 2020.

Projets de paysage 


\section{Le rôle des riverains dans le façonnement des interfaces ville- forêt}

Représentations et pratiques dans les franges urbaines des Landes de Gascogne

The Role of Local Inhabitants in Shaping City-Forest Interfaces Representations and Practises on the Urban Fringes of the Landes de Gascogne

Christine Bouisset et Isabelle Degrémont

1 L'objet de cet article est d'analyser les modes d'habiter des populations résidentes, au sens de résidence mais aussi de pratique des lieux (Hoyaux, 2000, 2003 ; Stock, 2004 ; Morel-Brochet, 2007), dans les lotissements périurbains au contact de la forêt des Landes de Gascogne. Si, depuis la création du massif forestier au xix ${ }^{e}$ siècle, la forêt de pins maritimes est devenue progressivement le cadre de vie ordinaire de la population rurale, la périurbanisation autour des grandes agglomérations et le long du littoral contribue maintenant à développer des zones de contact entre espaces urbanisés et espaces forestiers. Ce processus s'accompagne donc d'une réduction des surfaces forestières et d'une hausse de la fréquentation qui contribue à développer la fonction récréative de cette forêt majoritairement privée.

2 À ce titre, l'étude de cas du département des Landes apparaît intéressante. Hormis la partie littorale implantée sur les dunes, la forêt artificielle de pins maritimes est réputée laide et monotone tant au plan des paysages que de la biodiversité (Arnould $e t$ al., 2002; Boutefeu, 2007). De plus, cette forêt est soumise à un double processus d'urbanisation : celle des communes du littoral du fait d'une forte pression touristique et celle qui provient du développement des agglomérations de Dax, chef-lieu du département et surtout de Bayonne au Sud. Nous nous sommes donc interrogées sur les conséquences de la cohabitation des nouvelles populations urbaines et d'espaces forestiers jusque-là dédiés principalement à la production. Il s'est agi de comprendre le fonctionnement de ces franges urbaines: les représentations paysagères et les 
pratiques récréatives que les riverains ont de la forêt ainsi que les conséquences de cette proximité dans le façonnement des paysages d'interface. Alors que pour d'autres acteurs la forêt "plantée » des Landes n'est pas une "vraie » forêt, les habitants s'approprient un paysage et développent un discours construit et argumenté, une véritable rhétorique (Maciel, 2009) fondée sur leur expérience du quotidien (Gustafson, 2001 ; Tuan, 2006).

Un travail d'enquête par entretiens semi-directifs a été mené sur deux terrains auprès de plus de 60 résidents permanents en lisière forestière. Le premier terrain a été choisi dans le Sud des Landes : communes de Capbreton, Soorts-Hossegor, Labenne.

Figure 1. Carte de localisation des communes d'enquête

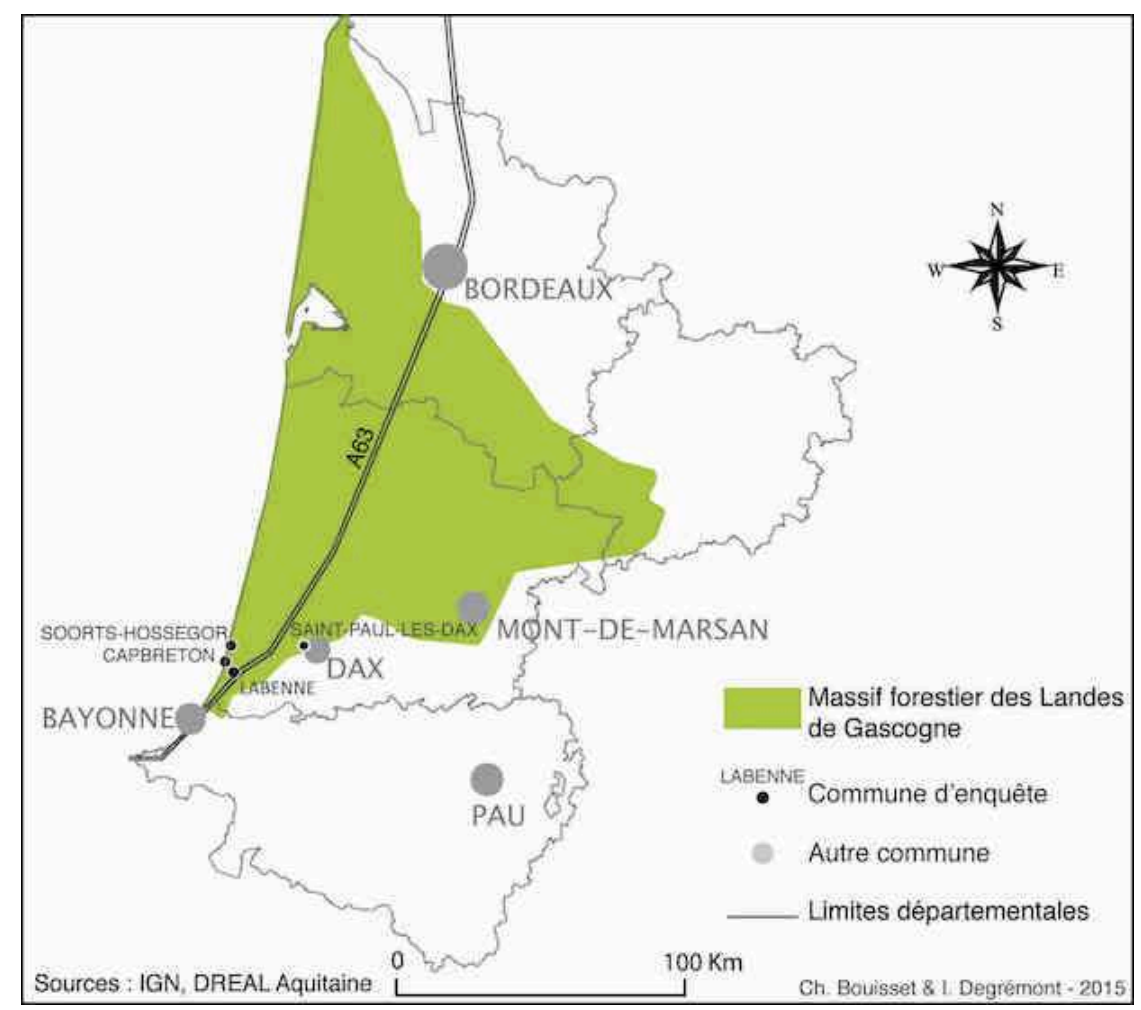

Sources: IGN, Dreal Aquitaine/Christine Bouisset et Isabelle Degrémont, 2015.

Proche de l'océan et de l'agglomération bayonnaise où les prix du foncier atteignent des sommets, il est soumis à une forte demande résidentielle et touristique. Le deuxième terrain, à la périphérie de Dax, se situe davantage à l'intérieur des terres. Il se caractérise par des processus périurbains plus classiques autour d'une ville moyenne, dans un espace où la fonction économique liée à l'exploitation du pin des Landes reste encore très importante. Le travail d'enquête s'est penché sur l'itinéraire résidentiel des habitants, leurs pratiques quotidiennes, leurs perceptions de la forêt environnante et la manière dont ils contribuent à l'aménagement de franges urbaines dont le façonnement résulte moins de projets urbains construits que de la juxtaposition d'initiatives individuelles à l'échelle microlocale. 


\section{La forêt comme voisine : hasard ou projet de vie?}

5 Les habitants interrogés résident principalement dans des maisons individuelles, dont ils sont pour la plupart propriétaires. Ils appartiennent majoritairement à des catégories socioprofessionnelles intermédiaires (employés, fonctionnaires) et, dans une moindre mesure, supérieures (cadres, chefs d'entreprises). Cette dernière catégorie est surtout présente dans la station touristique la plus ancienne et huppée: SoortsHossegor.

6 Les résidents enquêtés sont relativement âgés avec $60 \%$ de retraités qui se sont en général installés au moment de leur cessation d'activité. Les classes les plus jeunes (moins de 40 ans) avec des enfants en bas âge correspondent au deuxième groupe le plus présent dans l'échantillon. Ils sont cependant davantage localisés sur les communes littorales que sur l'agglomération dacquoise, notamment à Labenne et Capbreton qui ont développé une politique d'accession à la propriété en proposant des lotissements aux jeunes familles avec enfants originaires de la commune.

7 Concernant l'origine géographique, moins de $15 \%$ sont originaires de leur commune de résidence mais environ $60 \%$ sont originaires du Sud-Ouest, les $40 \%$ restants étant majoritairement originaires de la moitié nord de la France. Beaucoup occupent leur logement depuis longtemps: seuls $40 \%$ l'occupent depuis moins d'une décennie. Plus de $70 \%$ des habitants interrogés sont propriétaires et pour $7 \%$ le lieu de l'entretien est une résidence secondaire. La plupart des interrogés résident dans une maison avec jardin : plus de $87 \%$ contre moins de $13 \%$ pour les appartements (représentés surtout à Saint-Paul-lès-Dax qui a construit récemment des immeubles HLM au niveau de la dernière frange urbaine en lisière forestière). D'ailleurs, la proximité immédiate avec la forêt a été privilégiée dans le choix des zones d'enquête : il s'agit de maisons avec jardin ou d'immeubles intégrés la plupart du temps dans des lotissements en lisière forestière. Ces derniers datent pour les plus anciens des années 1960.

8 Les personnes interrogées ont toutes un discours très positif sur leur relation de voisinage à la forêt. Les mots fusent très vite pour qualifier ce rapport sentimental : "agréable », «joli », «calme » sont les principaux qualificatifs qui reviennent avec insistance. Mais quand on leur demande si les choix résidentiels ont été dictés par leur volonté de se retrouver « en forêt » ou " au voisinage » de la forêt, leur réponse montre une hiérarchie dans les critères ayant présidé à leur installation. Il faut ici distinguer les résidents venus pour leur retraite de ceux qui sont encore en activité.

Pour les premiers, la proximité de la forêt n'est pas mentionnée spontanément bien qu'elle fasse partie, très certainement, d'un «coup de cœur " général. En effet, un premier groupe explique que le choix de s'installer ici à la retraite provient de la découverte de la "région » pendant des vacances alors qu'ils étaient en activité. Le triptyque "océan-plage-forêt» qui a rendu célèbres certaines stations balnéaires comme Arcachon ou Hossegor, joue ainsi le rôle d'image de marque. De même, sans mentionner forcément la forêt, d'autres ont cherché, pour reprendre leurs expressions, un « environnement calme » et une « proximité » : ville/campagne, à la nature, à la mer (nous avons volontairement enlevé l'argument "proximité à la forêt» car probablement induit par la question posée). Même si plus lointaines, les Pyrénées sont aussi mentionnées dans l'intérêt d'un environnement plus "régional ». Ces types de mentions représentent plus de $15 \%$ des réponses données - qui montent à plus de $32 \%$ si l'on prend en supplément l'argument de la proximité avec la forêt. Certains retraités 
tablent davantage sur la situation en matière de commerces, de services et de la proximité (mais pas trop près non plus...) de grands centres urbains comme Bayonne ou Dax: ils décrivent alors longuement l'emplacement de l'habitation et sa situation. Quelques-uns ont également eu un «coup de cœur", non pas sur l'environnement voisin ni sur l'adresse et la situation du logement mais davantage sur sa qualité architecturale (maison en bois, maison avec grande terrasse, avec piscine, etc.). Ainsi, les choix résidentiels des retraités sont souvent le fruit d'un projet de vie qui intègre le choix de la région et, à l'échelle locale, la volonté de s'installer dans un entre-deux périurbain choisi pour ses aménités.

Les plus jeunes arrivés ainsi que les classes d'âge intermédiaire (30-50 ans) développent, quant à eux, un discours qui porte essentiellement sur des raisons professionnelles pour expliquer le choix résidentiel ( $14 \%$ des réponses) et la volonté de trouver un logement proche de la famille (14\% également). Viennent ensuite des arguments qui soulignent plutôt le fait que le logement est une propriété de famille (plus de $5 \%$ des réponses) ou bien qu'il s'agit d'une habitation dans un lotissement pour primo-accédants (moins de $3 \%$ des réponses). Beaucoup d'entre eux émettent également l'idée que, s'il s'agit de lieux de villégiature pour d'autres, ils sont sensibles eux-mêmes à cet « air de vacances ».

Figure 2. L'argument premier du choix résidentiel des enquêtés

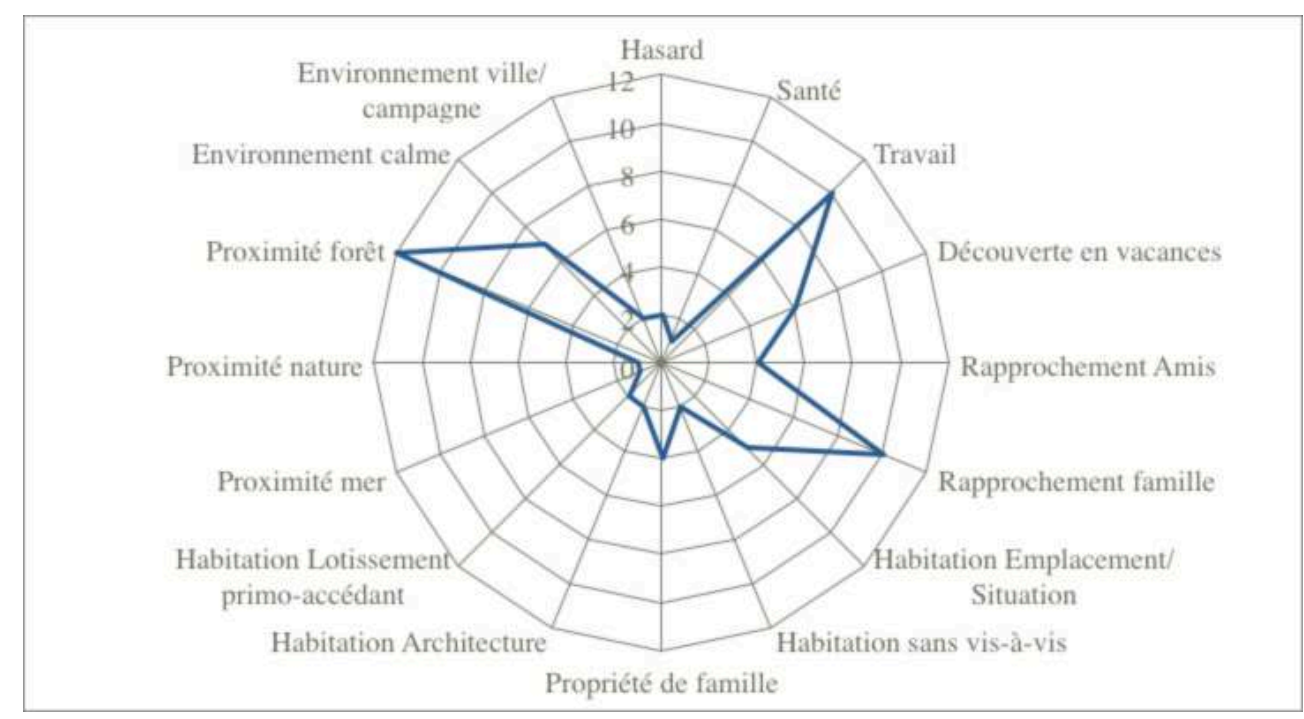

11 Dans tous les cas, plusieurs arguments successifs sont avancés par les enquêtés pour expliquer le choix résidentiel. Quand le sentiment d'« environnement naturel » et "forestier" n'a pas été donné en premier argument, il apparaît souvent ensuite. Comme dans d'autres espaces périurbains, les entretiens montrent ainsi que si les enquêtés n'ont pas nécessairement été guidés en premier lieu par le projet d'habiter " en forêt ", à l'usage, ils sont enchantés de ce cadre de vie, ils apprécient la tranquillité et la proximité de la nature (Benages-Albert et Bonin, 2013). Ils aiment « leur forêt » et sont capables de décrire et d'argumenter les valeurs qu'ils lui associent. En effet, alors même que la forêt est rarement le seul facteur déterminant dans les choix résidentiels, elle constitue un cadre de vie unanimement apprécié et est le support de nombreuses pratiques récréatives: balades à pied ou à vélo, promenade du chien, cueillette de champignons, ramassage des pignes de pin... 
Figure 3. Les pratiques récréatives en forêt mentionnées par les riverains (plusieurs réponses possibles)

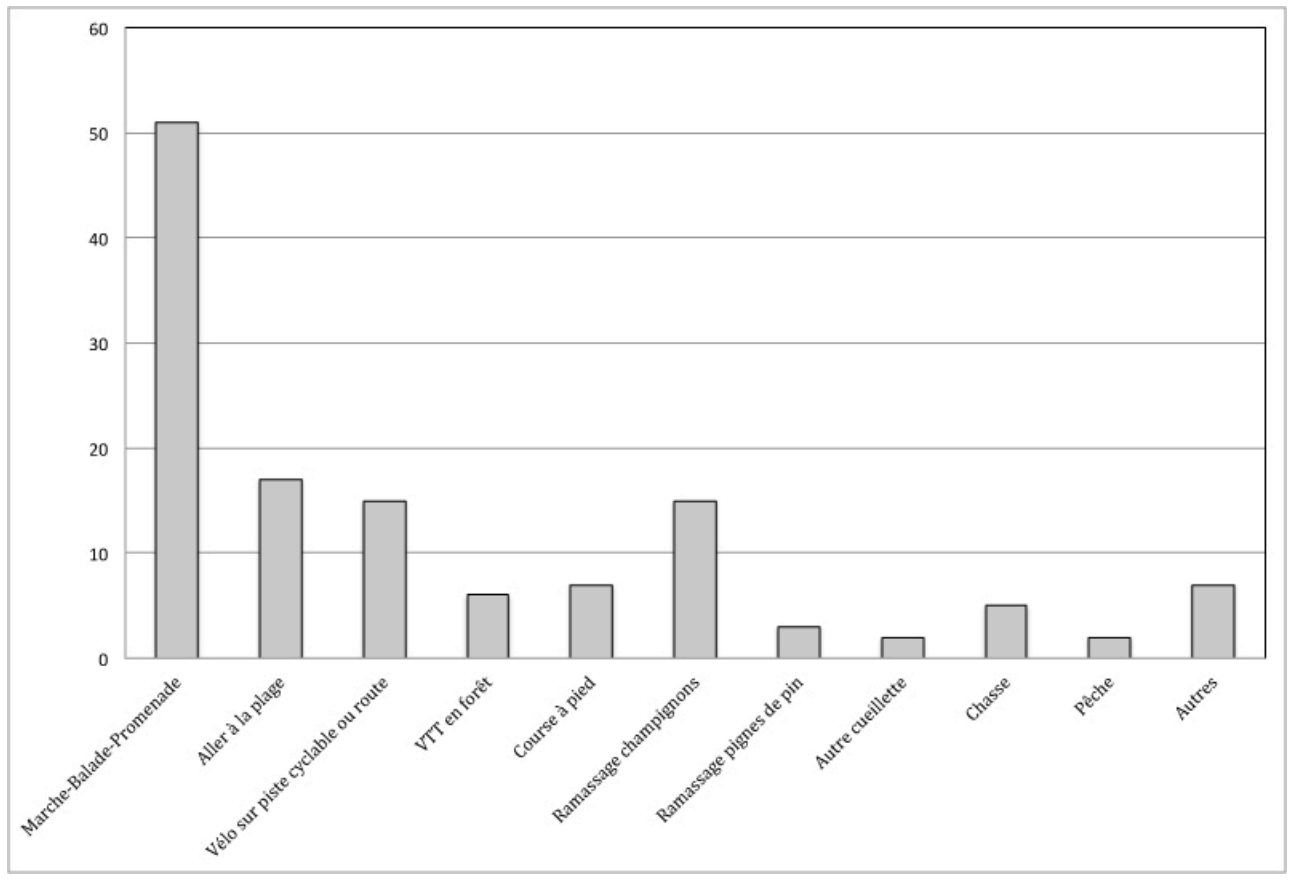

Les pratiques ont été longuement commentées par les enquêtés, qu'il s'agisse des plus anciens comme des plus récents résidents. Les activités récréatives « courtes" sont les plus couramment décrites car les habitants aiment avant tout se promener et profiter de la forêt sans prendre la voiture. Les promenades à pied ou à vélo sont donc les plus courantes. La marche, comme certains travaux le montrent déjà (par exemple, Roy, Paquette et Domon, 2005), suscite beaucoup d'enthousiasme et certains résidents vont se «balader ", se " promener ", « faire un tour ", tous les jours. Peu se promènent seuls, l'activité de sortie dans la forêt est donc une pratique en famille et avec les amis. Mais un certain nombre de personnes interrogées ne pratiquent pas ou plus l'espace forestier voisin. En revanche, ils observent tous la forêt depuis chez eux, par leur balcon ou par leur jardin.

\section{Profiter de la forêt au quotidien : pratiques récréatives et attributs paysagers}

L'objet de l'enquête, à travers des questions portant sur ce que les interviewés apprécient ou au contraire n'apprécient pas dans la forêt et sur ce qu'ils diraient pour la décrire à quelqu'un qui ne la connaît pas, était de mettre en relief le regard imprégné de valeurs (Droz et al., 2006) que les habitants portent sur les paysages environnants. Ce faisant, beaucoup de résidents décrivent d'abord la forêt "derrière » chez eux. Le devant est toujours considéré comme urbain et en contact avec les maisons et immeubles voisins. L'appropriation de la forêt est d'abord une appropriation visuelle depuis leur balcon, terrasse ou jardin. Cette dernière tend à dépasser les limites de la parcelle habitée stricto sensu : 
«On fait abstraction, si on peut dire, du grillage, et on a l'impression d'être propriétaires d'un grand espace boisé, etc., de derrière quoi, ce qui est tout de même très agréable, hein !» (E6, Labenne).

Certains vont même jusqu'à construire des terrasses pour contempler la forêt « de haut » et légèrement en retrait. On installe table et chaises pour profiter du panorama. En général, la vue permet d'observer les pins et le sous-bois ainsi que la végétation de lisière forestière. Selon la distance, certains cherchent également l'ombre des pins, qu'ils soient dans le jardin ou en lisière forestière.

Figure 4. Se ménager une vue sur la forêt (Saint-Paul-lès-Dax, Capbreton)

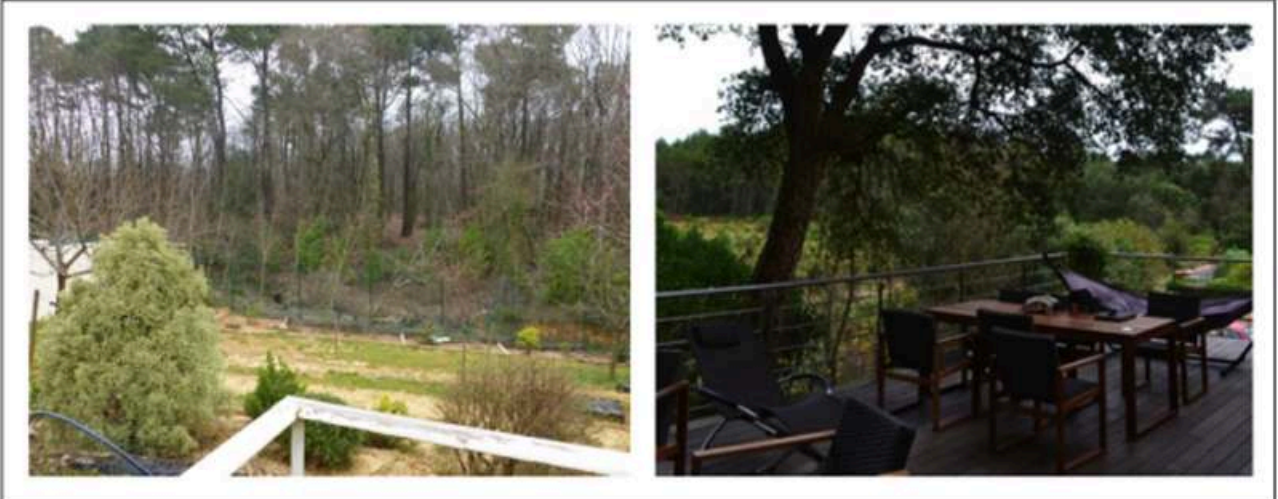

15 Une partie importante du discours sur le panorama visuel qu'offre la forêt porte sur l'évolution du paysage périurbain. Les vieux résidents, surtout, voient les espaces limitrophes s'urbaniser, souvent à leur grand désarroi : ils ne font pas forcément de remarques sur des problèmes de voisinage que cela induit mais ils regrettent de n'être plus suffisamment en contact avec la « nature » et surtout avec les animaux : oiseaux et chevreuils essentiellement. Les vues depuis les lotissements ont donc changé. Même s’il est parfois gardé un rideau d'arbres (surtout le cas de Saint-Paul-lès-Dax), les résidents voient maintenant de loin les nouvelles constructions. La forêt a tendance à devenir « haie » de séparation sur quelques mètres de profondeur.

Figure 5. Un mince rideau d'arbres face à l'urbanisation (Saint-Paul-lès-Dax)

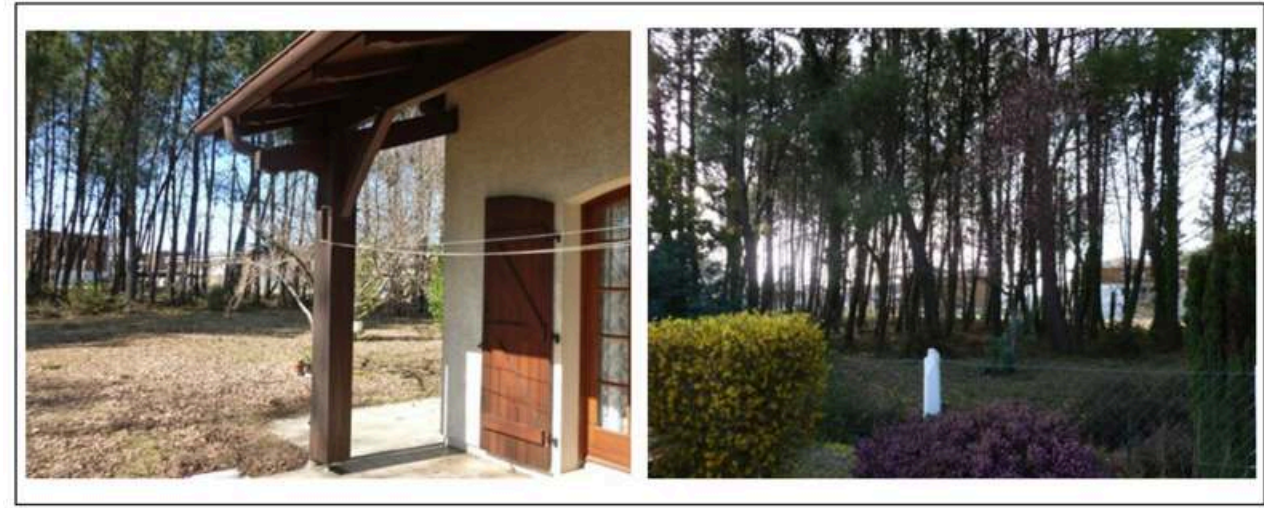

On note également que les surfaces nouvellement urbanisées sont, en général, totalement dépouillées des pins d'origine alors que les lotissements anciens étaient 
construits sous la forêt en conservant les arbres dans les parcelles, ce qui est déploré tant par les habitants anciennement installés que par les nouveaux résidents.

Figure 6. Lotissement récent construit après défrichement (Labenne)

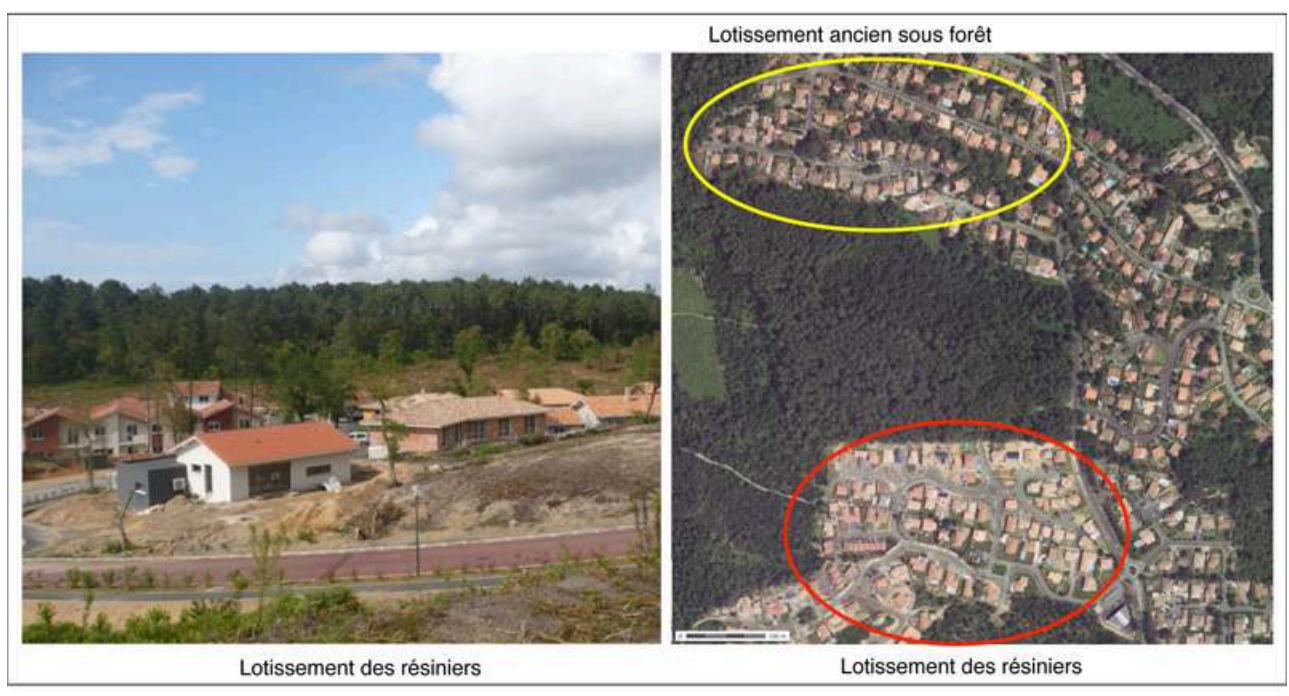

Sources : IGN, clichés Christine Bouisset, Isabelle Degrémont.

Le discours, à ce moment-là, développe l'idée que la forêt ne peut que valoriser la maison : la possibilité de la pratiquer, le sentiment d'avoir un plus grand terrain mais aussi le fait d'être sans vis-à-vis et de la contempler, de l'avoir pour soi finalement.

"C'est plus beau que d'avoir une maison en face. Je trouve ça magnifique, en plus ça vit. Là, l'été un peu moins, mais au printemps, il y a plein d'oiseaux qui viennent manger dans notre jardin, il y a des écureuils, c'est super-sympa. C'est sauvage [...] » (E18, Capbreton).

18 Le regard esthétique des riverains sur la forêt relève davantage du pittoresque que du sublime (Klingender, 1988) : contrairement aux images génériques de la forêt diffusées par la promotion touristique (Bouisset et Degrémont, 2015), on note l'existence d'un sentiment esthétique du quotidien, centré sur l'expérience spatiale microlocale des riverains, véritable "savoir-être" au lieu (Berdoulay et al., 2010). Leur discours a tendance à décomposer la forêt en différents attributs paysagers qui ne sont pas considérés de façon autonome mais sont la clé d'entrée pour comprendre le sentiment esthétique vis-à-vis de la forêt. Trois éléments composant le paysage forestier apparaissent de façon récurrente : une personnification du pin, la présence de la faune, des sous-bois clairs et variés changeant au fil des saisons.

19 La personnification du pin est fortement présente dans les discours des riverains. Les retraités ayant choisi leur résidence proche du littoral se focalisent en particulier sur la morphologie de l'arbre (fût droit, houppier haut) et sur l'aspect « toujours vert » quelle que soit la saison:

«Et vous voyez ça partout, même quand vous vous promenez en voiture, vous voyez d'un coup, un pin et le ciel bleu. C'est magnifique, je suis vraiment amoureuse de cette région » (E15, Capbreton).

20 Ainsi, si certains retraités sont bien conscients que c'est un arbre ordinaire qui se répète sur des kilomètres, ils lui trouvent, par sa morphologie, un côté exotique que l'on ne retrouve nulle part ailleurs : 
«[...] ça fait un peu exotique. Moi je trouve que des fois, quand on les regarde de loin, on se croirait avec des cocotiers, surtout quand ils ont été très abîmés, avec les branches en bas comme ça. Je disais souvent, "on est au pays des cocotiers" (sourire) » (E1, Capbreton).

21 Si les actifs sont moins sensibles à la « beauté » du pin que les retraités, ils ne sont pas en reste au niveau de l'élément symbolique qu'il peut représenter via notamment la " pigne de pin». Elle est ramassée par les adultes pour la cheminée, par les enfants pour les collectionner, on parle de "ses graines » pour la salade et même on se la fait tatouer pour affirmer son identité de « landais » (E27, Labenne).

La décomposition en éléments paysagers se poursuit ensuite à partir du sous-bois qui intéresse particulièrement les riverains : on observe essentiellement les mentions de la bruyère, des fougères, des genêts. Le désir d'être en contact avec la faune a également une forte résonance chez tous les enquêtés. Ce « besoin » relie les valeurs esthétiques et de bien-être. C'est peut-être là que le rapport avec la «nature » s'ancre le plus dans l'émotion: la beauté côtoie l'émerveillement devant le "vivant " non humain. Les habitants se sentent donc positivement entourés par une forêt vivante. C'est là également où le face-à-face monde urbain/nature est le plus flagrant. Ces habitants, la plupart d'origine urbaine, s'extasient devant une faune ordinaire, écureuils, oiseaux et leur chant, cigales, chevreuils... et s'émerveillent de l'expérience sensorielle offerte par la forêt à travers la vue, mais aussi les bruits et les odeurs. Leur discours rappelle qu'audelà du paysage, la «nature est un espace dynamique, en trois dimensions avec des qualités qui font appel à plusieurs sens » (Brady, 2007).

23 Le besoin de se promener dans une forêt claire est également bien mis en avant. Voir loin est apprécié pour un sentiment de sécurité et surtout pour le jeu de lumière esthétique que cela procure :

"C'est vrai que comme ce n'est pas très touffu, du coup on peut voir la lumière passer à travers. Le soir quand le soleil est bas, avant qu'il se couche derrière la maison, ça fait des taches orangées dans la forêt, c'est hyperbeau. C'est magnifique, on adore » (E18, Capbreton).

Cette perspective visuelle est également associée à l'idée que la forêt doit être entretenue et son accès facilité. À ce niveau, les résidents, même si la plupart ne sont pas capables de dire si la forêt voisine est communale, domaniale ou si elle appartient à des propriétaires privés, font une distinction entre la forêt à proximité des espaces urbains et littoraux et la forêt plus « intérieure ", celle que les automobilistes longent pour arriver à Bordeaux. Ils sont conscients que "leur » forêt voisine est davantage entretenue, moins sauvage, que le sous-bois peut être régulièrement éclairci pour des raisons, selon eux, essentiellement de loisirs :

« Pour se promener, c'est facile. Il n'y a pas besoin d'y aller à la machette. Vous avez des chemins partout. C'est très agréable de ce côté-là. Et puis c'est très agréable de se promener sous ces grands arbres » (E8, Labenne).

D'ailleurs, les discours convergent pour estimer que la forêt qu'ils côtoient n'est pas tout à fait la « vraie forêt » des Landes. Ils s'estiment tous en périphérie du massif et considèrent que «leur » forêt est soumise à une urbanisation qui change le paysage. Certains la qualifient non de forêt mais de «bois ». Ils distinguent ainsi deux forêts. La première, la forêt landaise « véritable » se localise plus au nord, plus au cœur du massif. Certains la nomment « Haute Lande » ou «Grande Lande ». Ils la décrivent comme plus «sauvage» en arguant d'une faible urbanisation mais aussi d'une plus grande importance de la faune, qui serait là moins dérangée par l'homme. Ils estiment aussi 
que les pins sont plus alignés et sont dédiés davantage à l'exploitation forestière que là où ils habitent. Leur forêt "périphérique ", par opposition, serait plus diversifiée dans les essences (pour le littoral) et beaucoup moins alignée (pour tous), plus humanisée par la présence de l'habitat et la fréquentation. Ce discours est identique pour tous les résidents enquêtés. Le constat de la séparation de la forêt en deux zones distinctes par les riverains les amène à commenter la réduction spatiale de la forêt en périphérie du massif. Spontanément, en abordant cette distinction, les riverains réfléchissent alors à la pérennité de la forêt et au fait qu'elle est peut-être en danger.

\section{Interfaces en projet : aménager et jouer avec les frontières}

Dans tous les cas d'interface, l'appropriation habitante est d'autant plus forte que les bois limitrophes des villes et villages ne sont pas systématiquement exploités par des propriétaires en attente d'opportunité foncière :

«Je vois, par exemple ici, je ne sais pas à qui ça appartient mais ce n'est jamais entretenu. On ne voit jamais personne aller dans la forêt »(E17, Capbreton).

Pour le riverain, la forêt de bordure a donc l'air à l'abandon, ce qui renforce le sentiment d'être face à un espace public. Cette appropriation est source de tensions quant au véritable statut du foncier forestier et aux usages développés par les propriétaires de parcelles urbaines et ceux des parcelles boisées. Elle témoigne d'un processus de patrimonialisation qui amène les habitants à considérer que la forêt voisine est un bien commun, un véritable espace public que l'on peut collectivement pratiquer sans se soucier de son statut foncier souvent privé.

Mais cette appropriation de la forêt par les riverains se heurte parfois aux projets des communes et au traitement des zones forestières dans les documents d'urbanisme: bien que majoritairement protégées car souvent classées en zones naturelles, les forêts sont en effet le support privilégié de l'extension de l'urbanisation en périphérie urbaine. En l'absence d'éléments naturels jugés "remarquables», la forêt de production elle-même est peu concernée par les dispositifs réglementaires de protection de la nature (Deuffic et al., 2010) et les PLU des communes étudiées considèrent tous que la plupart des paysages forestiers ne présentent pas d'intérêt particulier. Cette banalité, couplée à l'abondance de la forêt en terme d'occupation du sol, justifie qu'elle serve de réserve foncière (plus que les espaces agricoles, jugés davantage stratégiques). Cet état de fait est regretté par la quasi-totalité des personnes interviewées. Les riverains interrogés à Capbreton se plaignent par exemple des projets d'urbanisation (zones $\mathrm{AU}$ ) en périphérie de la ville ainsi que de l'achèvement d'un contournement routier dont l'emplacement est réservé au PLU, projets qui risquent à court ou à moyen terme de créer des nuisances, voire carrément de faire disparaître le cadre forestier qui les entoure. 
Figure 7. Les projets d'aménagement sur les franges urbaines inscrits au PLU de Capbreton

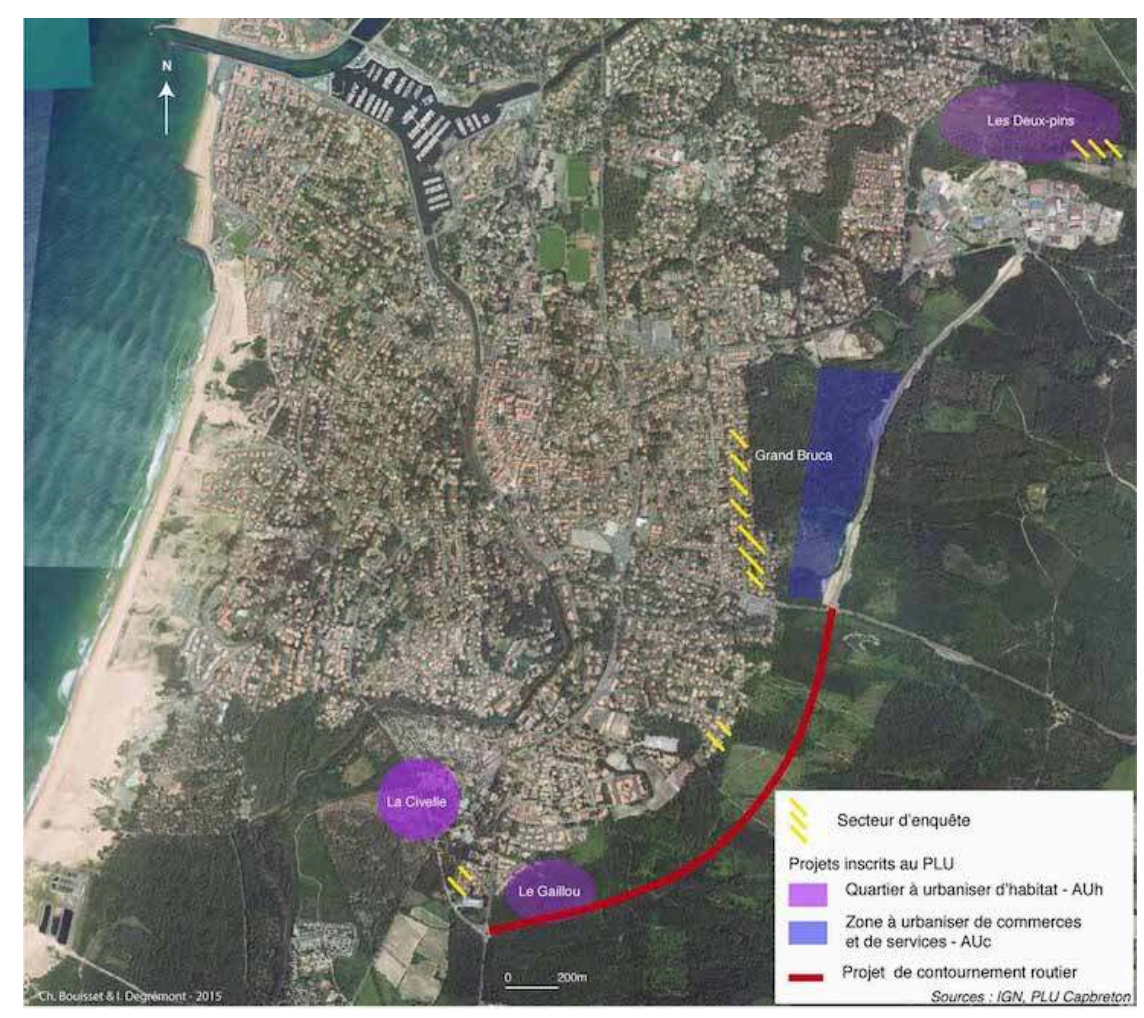

Sources : IGN, PLU de Capbreton.

29 L'emplacement de la «ligne » entre forêt et espace bâti est donc mouvant : il est principalement le fait d'extensions successives de l'urbanisation décidées à l'échelle communale. Mais la physionomie de ces franges urbaines à l'échelle micro relève davantage des projets individuels des riverains, via l'aménagement de leur propriété et de ses abords, que de projets publics d'aménagement. La pratique du jardin et de ses limites avec la forêt varie considérablement en fonction du rapport à la forêt et à la nature. On distingue plusieurs types de comportement chez les résidents en maison. Un premier groupe instaure une limite nette entre le jardin et la forêt au point que parfois les résidents se coupent visuellement du panorama forestier avec l'édification de haies végétales ou construites qui occultent la vue ( $40 \%$ des jardins observés). 


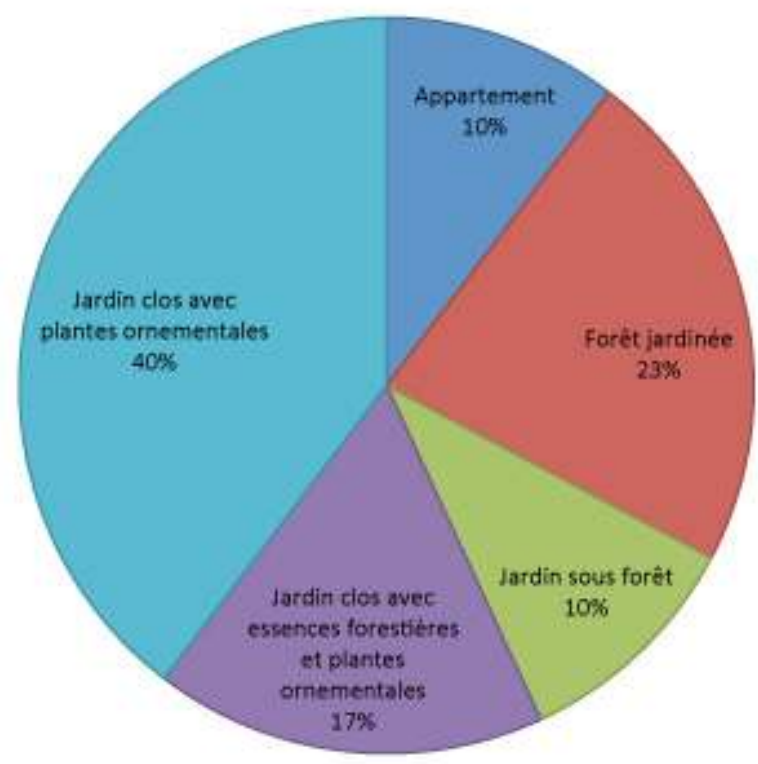

Cette limite permet de séparer une nature jardinée d'une nature sauvage que représente la forêt. La plupart du temps, ces jardins abritent des plantes d'ornement qui ne sont pas originaires du milieu landais ou plus rarement des arbres fruitiers. Ils possèdent tous des pelouses rases. Ce sont des jardins très aménagés: terrasses, bordures cimentées, arrosages automatiques, cabanes pour les outils, etc. Ils intègrent également beaucoup d'équipements de jeu pour enfants. Plus rarement, il s'agit de jardins-pelouses peu ornementés, quasi vides. Souvent, la haie est haute y compris du côté de la forêt.

Figure 9. Clôturer le jardin (Capbreton, Saint-Paul-lès-Dax)

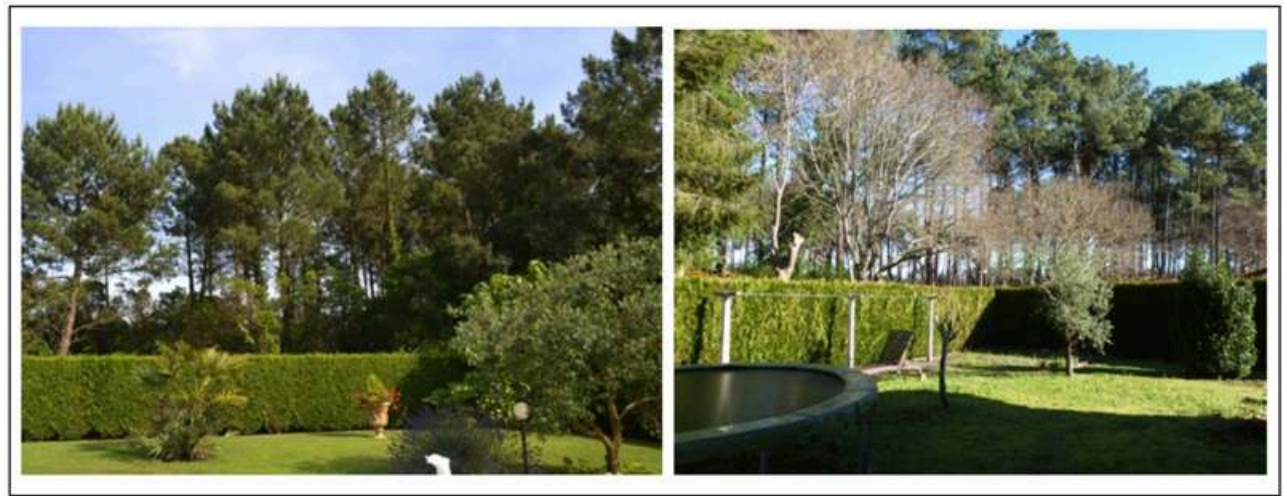

31 On ne peut donc pas voir le sous-bois, seuls les houppiers des pins dépassent de la haie. Les raisons parfois invoquées à cette occultation volontaire portent sur le besoin de se sentir chez soi (Gross et Lane, 2007), sans être vu par quiconque :

«J'aurais aimé qu'il y ait plein d'arbres partout. Que ça fasse beaucoup plus... Qu'on soit complètement envahi d'arbres... envahi, pas forcément au milieu, mais sur les bords. [...] Le truc marron là (un brise-vue en plastique marron adossé au grillage), 
on vient de le mettre parce que la haie ne pousse pas assez vite. Les gens voyaient tout ce qu'on faisait, tout le monde me dit "je t'ai vu dans ton jardin". On n'a pas de chez nous » (E5, Labenne).

\section{Ce premier group
portail d'accès.}

Figure 10. Portes sur la forêt (Saint-Paul-lès-Dax)

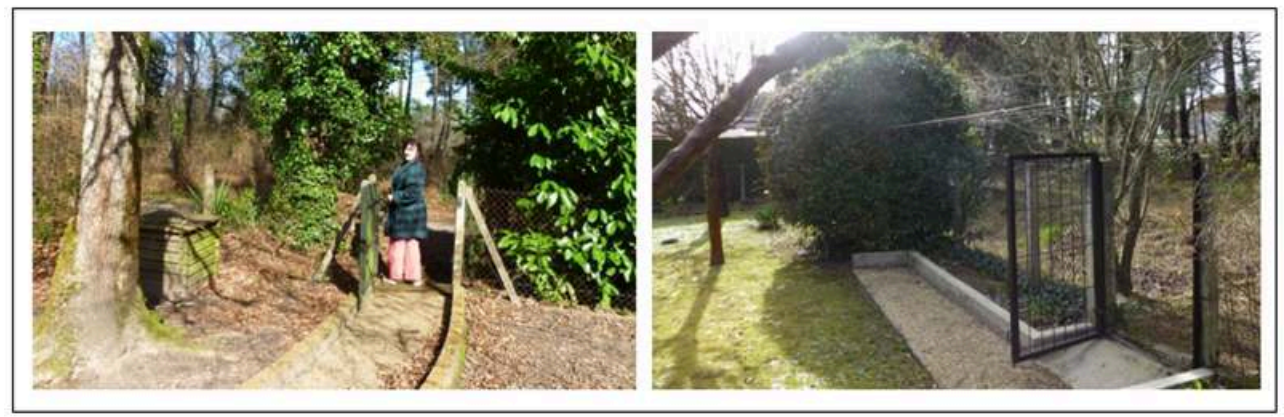

Mais la plupart du temps, les aménagements de portails sur la forêt sont le reflet d'un second type de jardin. Ce dernier est plus «ouvert " sur la forêt: s'il possède une frontière nette, celle-ci est composée de grillage et n'occulte pas la vue. Dans ce groupe, deux attitudes prévalent en matière de jardin (et de pratiques). Une attitude où le jardin est très aménagé, à l'instar du premier groupe : cheminements en dalle, grillages avec murette, bordures cimentées fleuries. Ou, à l'inverse, on observe des jardins davantage "nature" où les arbres tels que le chêne ou le pin ainsi que parfois une strate arbustive (arbousiers, genêts) sont présents. En général, ces derniers jardins n'ont pas de pelouse plantée. Ils se rapprochent davantage de la forêt (17 \% des jardins). Les habitants de ce genre de jardin revendiquent d'ailleurs le parallèle, même si cela leur occasionne parfois des problèmes de voisinage urbain :

«Chez moi, c'est resté un peu sauvage, j'aime bien, je me ressource dans mon "fourasta". Il y a des gens qui ne comprennent pas pourquoi je n'ai pas tout pelé, fait comme les gens d'à côté. Ils ont tout pelé alors qu'ils avaient comme moi, que des arbres. Ils ont enlevé tous les arbres. Ils ne supportent pas quand il y a une feuille... » (E12, Labenne).

On constate souvent ce libre "accès » à la forêt par des portails à Saint-Paul-les-Dax comme si l'ambiance était davantage campagnarde : les jardins donnent l'impression d'être plus tournés sur l'extérieur que ceux du littoral qui sont peut-être le reflet d'un repli urbain plus individuel. En revanche, cette attitude, si elle existe, n'apparaît pas dans les discours des enquêtés, qu'ils soient sur le littoral ou à Saint-Paul-les-Dax.

Enfin, on note un troisième groupe de jardins, celui qui est le plus en contact avec la forêt. Il ne possède pas toujours de frontières matérielles avec cette dernière. Il n'existe donc pas de limites nettes entre la fin du jardin et le début de la forêt. On oscille entre un jardin en forêt ou une forêt jardinée (respectivement 10 et $23 \%$ des jardins observés). Dans ce dernier cas, le jardin s'est installé sous les pins qui sont les seuls grands arbres du jardin, décorés avec des fleurs et des arbustes à leur pied. 
Figure 11. Forêt jardinée (Labenne, Saint-Paul-lès-Dax)

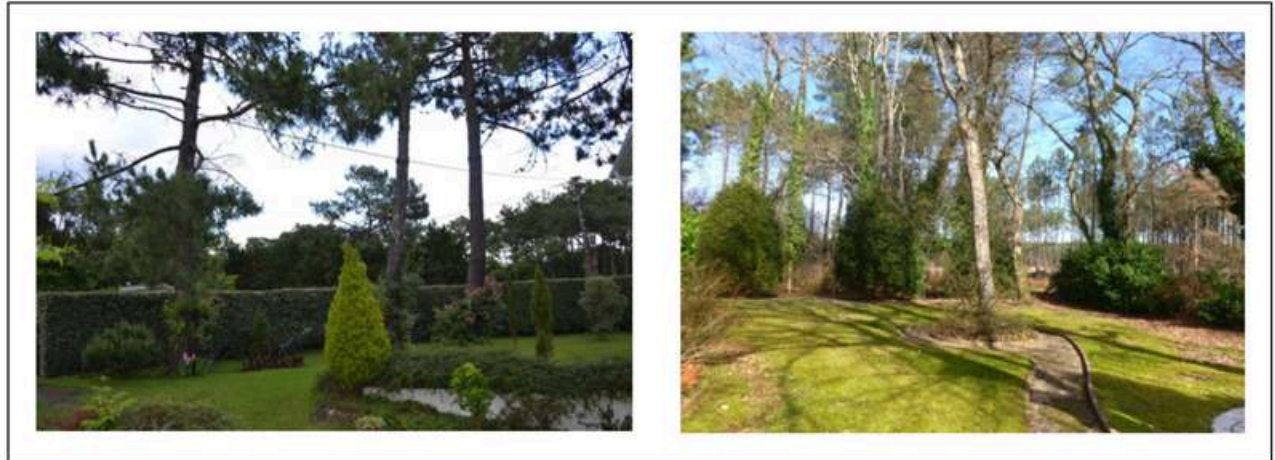

Cette forêt jardinée est très entretenue avec souvent une pelouse plantée et en général arrosée régulièrement. Certains de ces jardins, plus naturels, ont moins de pelouses à découvert et un sous-bois forestier plus développé : fougères, genêts, bruyères mais sans une grande densité d'arbustes. Il s'agit dans tous les cas de jardins ombragés avec une densité de pins importante. Toujours dans ce groupe où forêt et jardin s'entremêlent, d'autres propriétés ressemblent davantage à des jardins en forêt.

Figure 12. Jardins en forêt

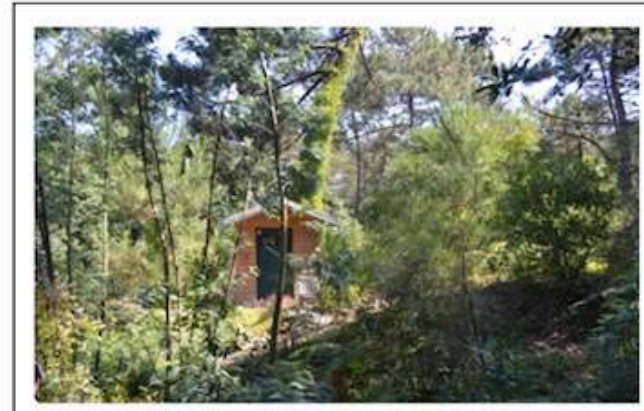

Capbreton, Allée du Grand Burca

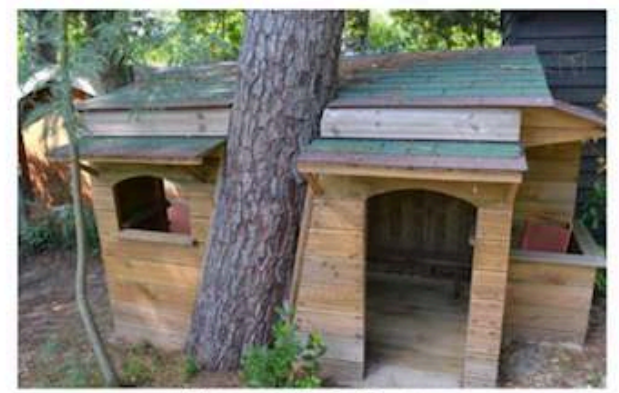

Labenne, Allée de Bourgogne

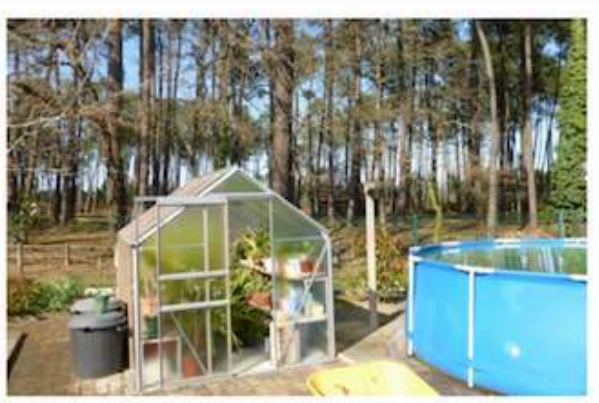

Saint-Paul-les-Dax,Av. Allende

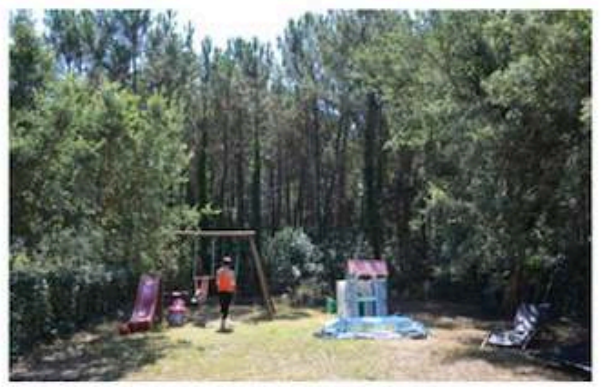

Capbreton, Lotissement les Genêts

37 Il s'agit souvent d'installer des équipements : abri de jardin, piscine, cabanon pour le bois ou pour la cuisine extérieure, aire de jeu. Les pins mais aussi une strate arbustive sont très présents en limite de propriété et entourent littéralement l'endroit où se concentrent les équipements. Les résidents jouent alors avec les arbres et les arbustes : ne voulant pas les abattre, ils déploient des trésors d'ingéniosité pour aménager le jardin : cabane d'enfant autour d'un pin, corde à linge sur supports «naturels »... Les maisons se retrouvent donc sous une épaisse végétation. 
surprise, c'est dans le second et troisième groupe de jardins que l'on retrouve les discours les plus dithyrambiques sur le contact avec la nature et tout particulièrement avec les animaux de la forêt. Les habitants possèdent une «culture » de la dynamique du milieu dans lequel ils vivent véritablement. C'est là aussi que l'on trouve les riverains qui parlent de leur jardin en terme de « bois » :

«(Mme) Il faut entretenir. On prend un sécateur, on monte, on descend... on coupe toutes les tiges qui poussent.

«(M.) Nous, on a un bois qui est propre. Enfin un bois, c'est petit. [...] Il y a une quinzaine de pins »(E11, Soorts-Hossegor).

Mais il arrive aussi que les pratiques des riverains débordent et annexent la forêt voisine. Le jeu le plus courant consiste à se servir de la lisière forestière pour entreposer son bois de chauffage. Le volume stocké par rapport à la taille des jardins explique très certainement ce genre de comportement. On note également que certains utilisent la zone forestière pour entreposer le compost ou autres débris végétaux du jardin, voire y installer un brasero. La forêt sert donc d'annexe utilitaire. Si tous ces comportements révèlent un manque de place et une volonté de ne pas s'encombrer, d'autres appropriations de la forêt sont nettement plus esthétiques : on aménage "sa " forêt voisine à son goût avec la plantation d'essences arbustives d'ornement, de massifs de fleurs, parfois de pelouse, en s'affranchissant des limites de propriété. Les pratiques des riverains sont donc souvent en adéquation avec leurs valeurs esthétiques et de bien-être vis-à-vis de la forêt :

«On a beaucoup de chênes-lièges ici. D'ailleurs, c'est un arbre qu'on aime beaucoup nous. Je les entretiens exprès là-bas, ce n'est pas chez nous mais j'entretiens » (E30, Capbreton).

Les limites boisées se "déplacent " également en raison du contexte des tempêtes de 1999 et 2009. Certaines parcelles urbaines "sous forêt ", abritant des pins âgés, ont été fortement endommagées. Les riverains ont découvert que le pin est un arbre qui ne supporte pas les grands coups de vent et peut casser net. Certains habitants, par peur ou par anticipation de nouvelles tempêtes, ont alors coupé les arbres les plus proches des maisons. Cela donne alors des jardins moins forestiers avec l'apparition de pelouses vertes et nues à l'abord immédiat des maisons. Le pin est repoussé en périphérie de la propriété pour des raisons de sécurité mais aussi de confort :

« Il y en avait deux dans le coin là-bas, mais avec le voisin, à cause de sa piscine, ça devenait dangereux. Donc, on en a abattu deux. Et là aussi, il y en avait deux, mais c'est nous qui les avons enlevés quand on est arrivés à la retraite, parce que le soleil se lève là, et comme notre maison est plein nord, on avait jamais le soleil. On ne pouvait jamais se mettre dessous, à cause de la résine, donc... » (E13, Labenne).

Mais pour autant, même chez eux, l'arbre en lui-même et la forêt ne sont pas rejetés : on apprécie au contraire encore plus de les voir et de profiter de la forêt... autour de chez soi.

\section{Conclusion}

Les riverains considèrent positivement le fait d'habiter dans un entre-deux périurbain : ni tout à fait en centre-ville ni tout à fait dans des espaces naturels «sauvages ». Il s'agit d'une pratique urbaine et quotidienne de la forêt, où la contemplation, la promenade et les loisirs sont privilégiés. L'échelle de pratique est réellement microlocale car l'intérêt de l'habitant est de profiter de la forêt comme un espace de

Projets de paysage, 13 | 2015 
voisinage sans prendre la voiture. Si l'installation ne résulte pas toujours d'un projet de vie intégrant initialement la forêt, le cadre périurbain est incontestablement apprécié et la proximité induit une façon de vivre avec la forêt, notamment dans la manière dont les habitants pratiquent le voisinage entre le jardin et la forêt. Certains ne font que contempler la forêt voisine depuis leurs fenêtres ou leur terrasse mais développent un attachement fort à cette vue. En revanche, beaucoup pratiquent la forêt, se l'approprient par des activités de loisirs et s'y projettent en la considérant comme une extension de leur jardin. Ceux-là possèdent souvent des portes de jardin qui donnent directement accès à la forêt. Ils jardinent "leur " forêt même s'ils n'en sont pas propriétaires ou bien ils «naturalisent » leur bois-jardin à l'image de la forêt voisine contribuant au façonnement de franges urbaines où les pouvoirs publics interviennent peu.

Ainsi, les paysages forestiers ordinaires participent bien à un processus de construction identitaire (Bigando, 2008) qui inclut ici une véritable dimension esthétique s'exprimant tant "dehors" via l'appréciation de certaines composantes du paysage forestier, que "dedans " à travers l'aménagement des jardins et la façon dont les habitants jouent sur la présence des arbres. Cette approche sensible confirme la place de l'esthétique comme une dimension centrale des relations entre sociétés et environnement (Sgard, 2010) quand bien même les acteurs de la protection de la nature et ceux de l'aménagement que sont certaines communes, surtout soucieuses de développer leur offre de logement, ne voient dans ces forêts artificielles que des paysages sans intérêt et des opportunités foncières.

Si l'ampleur de la demande d'un «cadre de vie forestier » est forte, c'est aussi que la forêt qui jouxte les lotissements est pratiquée, par les riverains, comme un véritable espace public où le propriétaire forestier est d'autant moins connu que la forêt est ouverte, sans clôtures et limites de propriétés. La forêt apparaît comme un lieu investi de valeurs, comme un patrimoine, non pas institué mais fruit d'un processus spontané porté par des individus (Riegl, 1903; Söderström, 1987; Rautenberg, 2003). Les riverains confirment par là même que le patrimoine définit moins ce que l'on possède que ce que l'on est (Hartog, 2003). Ce registre des valeurs " habitantes», fondées sur l'expérience des lieux (Tuan, 2006; Paris, 2011), construit donc un patrimoine du quotidien, banal, dédié à la jouissance d'un cadre de vie en contact avec la «nature » qui diffère fondamentalement des discours et critères de jugements officiels sur les espaces ou les paysages "à protéger », lesquels insistent davantage sur les qualificatifs de singularité, de rareté, de biodiversité, etc. (Bouisset et Degrémont, 2013). Pour les riverains, l'enjeu est d'autant plus important que ce n'est que dans ces paysages du quotidien, via leurs pratiques récréatives et le jardinage, que beaucoup peuvent communiquer avec la nature (Brady, 2007). Dans cette logique patrimoniale, ils sont d'autant plus attentifs à la présence forestière « dans » et « au bout » de leur jardin, que la plupart s'interrogent sur sa pérennité face aux phénomènes d'urbanisation. Ainsi, la grande crainte des riverains se cristallise autour des projets d'urbanisation où les communes sont considérées comme les responsables principaux de la déforestation. La conséquence de cette patrimonialisation est alors la naissance de mobilisations pour se préserver d'une urbanisation à laquelle ils ont, paradoxalement, eux-mêmes contribué. 


\section{BIBLIOGRAPHIE}

Arnould, P., Marty, P. et Simon, L., « Deux siècles d'aménagements forestiers : trois situations aux marges méridionales de la France », Ería, n 58, 2002, p. 251-267.

Benages-Albert, M., Bonin, S., « Le rapport au paysage ordinaire. Approche par les pratiques des espaces de proximité » Projets de paysage, $n^{\circ}$ 9, décembre 2013, URL : http:// www.projetsdepaysage.fr/fr/

le_rapport_au_paysage_ordinaire._approche_par_les_pratiques_des_espaces_de_proximite.

Berdoulay, V., Degrémont, I et Laplace-Treyture, D., « Savoir-être au lieu et mise en patrimoine de la nature dans les stations thermales des Pyrénées ( $\mathrm{XIX}^{\mathrm{e}}-\mathrm{XX}^{\mathrm{e}} \mathrm{s}$.) », Géographie et Cultures, $\mathrm{n}^{\mathrm{o}} 73$, Image et espace public, 2010, p. 39-62.

Bigando, E., « Le paysage ordinaire, porteur d'une identité habitante. Pour penser autrement la relation des habitants au paysage ", Projets de paysage, n 1, 2008, URL : http:// www.projetsdepaysage.fr/fr/le_paysage_ordinaire_porteur_d_une_identite_habitante.

Bouisset, C., Degrémont, I., « From communication to public perceptions : diversity of roles and values in a planted forest. The case of the landes of gascony (France) », XIV World forestry congress, Durban, South Africa, 2015.

Bouisset, C., Degrémont, I., « Construire un patrimoine naturel : valeurs (de société) contre critères (officiels) ? L'exemple de hauts lieux montagnards pyrénéens », Vertigo, hors série 16, Patrimonialiser la nature, 2013, URL : http://vertigo.revues.org/13750.

Boutefeu B., « La forêt comme un théâtre ou les conditions d'une mise en scène réussie ", thèse de doctorat en géographie, École normale supérieure lettres et sciences humaines-ENS-LSH Lyon, 2007.

Brady, E., « Vers une véritable esthétique de l'environnement : l'élimination des frontières et des oppositions dans l'expérience esthétique du paysage, Cosmopolitiques, $\mathrm{n}^{\circ}$ 15, 2007, p. 65-76.

Deuffic, P., Ginelli, L., Petit, K., « Les propriétaires forestiers face à l'écologisation des Landes de Gascogne : patrimoine foncier... et naturel ? ", Sud-Ouest européen, $\mathrm{n}^{\circ}$ 30, 2010, p. 109-123.

Droz, Y., Miéville-Ott, V., Spichiger, R. et Forney, J., « Le champ du paysage. Représentations paysagères et processus de légitimation des usages sociaux du paysage. De la vue-des-alpes au pays-d'en haut ", rapport de recherche, 2006, $67 \mathrm{p}$.

Gross, H., Lane, N., « Landscapes of the lifespan : Exploring accounts of own gardens and gardening ", Journal of Environmental Psychology, vol. 27, n 3, 2007, p. 225-241.

Gustafson, P., « Meanings of place : Everyday experience and theoretical conceptualizations », Journal of Environmental Psychology, vol. 21, n 1, 2001, p. 5-16.

Hartog, F., Régimes d'historicité, Paris, Éditions du Seuil, 2003, 272 p.

Hoyaux, A.-F., « Les constructions des mondes de l'habitant : Eclairage pragmatique et herméneutique ", Cybergeo. Revue européenne de géographie, janvier 2003, URL : http:// cybergeo.revues.org/3401.

Hoyaux, A.-F., « Habiter la ville et la montagne : essai de géographie phénoménologique sur les relations des habitants au Lieu, à l'espace et au territoire (exemple de Grenoble et Chambéry) », thèse de géographie, sous la dir. de Bernard Debarbieux, université Grenoble 1, 2000, 695 p. https://halshs.archives-ouvertes.fr/tel-00007867/. 
Klingender, F., « Le sublime et le pittoresque », Actes de la recherche en sciences sociales, vol. 75, 1988, p. 2-13.

Maciel, C.A.A., « Rhétorique du paysage et identité territoriales » dans Grandjean, P. (dir.), Construction identitaire et espace, Paris, L’Harmattan, coll. « Géographie et Cultures », 2009, p. 153-176.

Morel-Brochet, A., « À la recherche des spécificités du mode d'habiter périurbain dans les représentations et les sensibilités habitantes ", Norois, $n^{\circ} 205$, vol. 4, 2007, décembre 2009, http:// norois.revues.org/1237.

Paris, M., « Le végétal donneur d'ambiances : jardiner les abords de l'habitat en ville », thèse d'urbanisme mention architecture, sous la dir. de Balaÿ, O. et Fiori, S., université de Grenoble, 2011, vol. 1, 502 p.

Rautenberg, M., La Rupture patrimoniale, éditions À la croisée, coll. « Ambiances, ambiance », 2003, $173 \mathrm{p}$.

Riegl, A., Le Culte moderne des monuments. Son essence et sa genèse (1903), Paris, Éditions du Seuil, coll. « Espacements », 1984, $122 \mathrm{p}$.

Roy, L., Paquette, S., Domon, G., « La campagne des néoruraux : motifs de migration, territoires valorisés et usages de l'espace domestique », Recherches sociographiques, vol. 46, nº 1, 2005, p. 35-65.

Sgard, A., « Le paysage dans l'action publique : du patrimoine au bien commun », Développement durable et territoires, vol. 1, $\mathrm{n}^{\circ}$ 2, septembre 2010, URL : http://developpementdurable.revues.org/ 8565.

Söderström, O., «Logiques et pratiques ordinaires du patrimoine ", dans Zanetto, G. (dir.), Les Langages des représentations géographiques, Venise, Università Degli Studi Di Venezia, Dipartimento Di Scienze Economiche, 1987, p. 63-80.

Stock M., «L'habiter comme pratique des lieux géographiques », EspacesTemps.net, rubrique « Travaux », 2004, URL : http://www.espacestemps.net/articles/habiter-comme-pratique-deslieux-geographiques/.

Tuan Y.-F., Espace et lieu, la perspective de l'expérience (1977), Gollion, Infolio Éditions, coll. « Archigraphy Paysages », 2006, 219 p.

\section{NOTES}

1. Fourré, bois, terre inculte et couverte de broussailles, en occitan (Gascogne).

\section{RÉSUMÉS}

L'objet de cet article est d'analyser les modes d'habiter des populations résidentes dans les lotissements périurbains au contact de la forêt des Landes de Gascogne. Il s'agit, à partir d'un travail d'enquête, de comprendre le fonctionnement de ces franges urbaines : les représentations 
paysagères et les pratiques récréatives que les riverains ont de la forêt ainsi que les conséquences de cette proximité dans le façonnement des paysages d'interface. Alors même que la forêt n'est pas toujours un facteur prépondérant dans les choix résidentiels initiaux, le discours des habitants relève de valeurs esthétiques émotionnelles qui témoignent de la force de certains usages et fonctions attribués à la forêt, si artificielle soit-elle. Ces valeurs s'expriment via l'appréciation de certaines composantes du paysage forestier et à travers l'aménagement du cadre de vie dans des interfaces dont le façonnement relève plus d'initiatives individuelles que de projets collectifs.

The aim of this article is to analyse the ways in which resident populations inhabit suburban plots of land on the fringes of the forest of the Landes de Gascogne. The results of a survey are used to understand the way these urban fringes function: the representations of local inhabitants of the forest landscape and the recreational activities they practise in the forest as well as the consequences of this proximity on the way these landscape interfaces are shaped. Whereas the forest is not always among prevalent factors in initial residential choices, the discourse of the inhabitants relates to emotional aesthetic values which bear witness to the importance of certain uses and functions attributed to the forest, however artificial this landscape may be. These values are expressed via the appreciation of certain components of the forest landscape and through the development of the living environment in interfaces the shaping of which is more often the result of individual initiatives than that of community projects.

\section{INDEX}

Mots-clés : habitants, forêt, périurbanisation, interface, paysage

Keywords : inhabitants, forest, peri-urbanisation, interface, landscape

\section{AUTEURS}

\section{CHRISTINE BOUISSET}

Christine Bouisset est maîtres de conférences en géographie, université de Pau et des Pays de l'Adour, Laboratoire SET, UMR CNRS 5603.

christine.bouisset[at]univ-pau[dot]fr

\section{ISABELLE DEGRÉMONT}

Isabelle Degrémont est maîtres de conférences en géographie, université de Pau et des Pays de l'Adour, Laboratoire SET, UMR CNRS 5603.

isabelle.degremont[at]univ-pau[dot]fr 